\title{
Unveiling the Redox Control of Plant Reproductive Development during Abiotic Stress
}

\section{OPEN ACCESS}

Edited by:

Mohammad Anwar Hossain, Bangladesh Agricultural University,

Bangladesh

Reviewed by:

Girdhar Kumar Pandey,

University of Delhi, India

Gábor Kocsy,

Centre for Agricultural Research,

Hungary

Vivekanand Tiwari,

Weizmann Institute of Science, Israe

*Correspondence:

Gaurav Zinta

gzinta@gmail.com:

Asif Khan

asif.khan@cos.uni-heidelberg.de

${ }^{\dagger}$ Present Address:

Gaurav Zinta and Vipasha Verma,

Shanghai Center for Plant Stress

Biology, Shanghai Institutes of

Biological Sciences, Chinese

Academy of Science, Shanghai, China

${ }^{\ddagger}$ Equal first authors.

Specialty section:

This article was submitted to

Plant Biotechnology,

a section of the journal

Frontiers in Plant Science

Received: 07 March 2016

Accepted: 06 May 2016

Published: 16 June 2016

Citation:

Zinta G, Khan A, AbdElgawad H,

Verma V and Srivastava AK (2016)

Unveiling the Redox Control of Plant

Reproductive Development during Abiotic Stress. Front. Plant Sci. 7:700.

doi: 10.3389/fpls.2016.00700

\author{
Gaurav Zinta ${ }^{1,2 * \neq}$, Asif Khan ${ }^{3 *}$, Hamada AbdElgawad ${ }^{2,4}$, Vipasha Verma ${ }^{5 \dagger}$ and \\ Ashish Kumar Srivastava ${ }^{6}$ \\ ${ }^{1}$ Centre of Excellence Plant and Vegetation Ecology, Department of Biology, University of Antwerp, Antwerp, Belgium, \\ ${ }^{2}$ Integrated Molecular Plant Physiology Research, Department of Biology, University of Antwerp, Antwerp, Belgium, \\ ${ }^{3}$ Research Group Germline Biology, Centre for Organismal Studies Heidelberg, University of Heidelberg, Heidelberg, \\ Germany, ${ }^{4}$ Department of Botany, Faculty of Science, University of Beni-Suef, Beni-Suef, Egypt, ${ }^{5}$ Department of \\ Biotechnology, Dr Y S Parmar University of Horticulture and Forestry, Solan, India, ${ }^{6}$ Nuclear Agriculture and Biotechnology \\ Division, Bhabha Atomic Research Centre, Mumbai, India
}

Plants being sessile in nature are often challenged to various abiotic stresses including temperature fluctuations, water supply, salinity, and nutrient availability. Exposure of plants to such environmental perturbations result in the formation of reactive oxygen species (ROS) in cells. To scavenge ROS, enzymatic and molecular antioxidants are produced at a cellular level. ROS act as a signaling entity at lower concentrations maintaining normal growth and development, but if their levels increase beyond certain threshold, they produce toxic effects in plants. Some developmental stages, such as development of reproductive organs are more sensitive to abiotic stress than other stages of growth. As success of plant reproductive development is directly correlated with grain yield, stresses coinciding with reproductive phase results in the higher yield losses. In this article, we summarize the redox control of plant reproductive development, and elaborate how redox homeostasis is compromised during abiotic stress exposure. We highlight why more emphasis should be given to understand redox control of plant reproductive organ development during abiotic stress exposure-to engineer crops with better crop yield. We specifically discuss the role of ROS as a signaling molecule and its cross-talk with other signaling molecules such as hormones and sugars.

Keywords: oxidative stress, antioxidants, sexual reproduction, anther, gynoecium, pollen

\section{INTRODUCTION}

The entire life cycle of flowering plants is a succession of distinct growth phases, where plants depict various developmental stages. The growth phase between seed germination and vegetative maturity is termed as "vegetative-phase"; and the subsequent phase including formation of reproductive organs, sexual reproduction, and seed set is termed as "reproductive-phase." Plant performance in these growth phases, and transition from vegetative to reproductive phase is under tight control of genetic network (Huijser and Schmid, 2011). Moreover, signaling mediators such as reactive oxygen species (ROS), reactive nitrogen species (RNS), calcium, and phytohormones play crucial roles in integrating information from various endogenous and environmental cues, thus regulating plant growth and developmental transitions (Kocsy et al., 2013; Considine and Foyer, 2014; Traverso et al., 2014). Of these, ROS or redox mediated signaling has recently emerged as a core signaling 
pathway that shows crosstalk with calcium (Steinhorst and Kudla, 2013; Gilroy et al., 2014) and hormone-mediated signaling (Bartoli et al., 2013; Xia et al., 2015). The redox state is a broad term often described as an integrated ratio of different redox couples present inside the cell (König et al., 2012). Each developmental stage possess a specific redox pattern, determined by the concerted action of various ROS producing [NADPH oxidase (NOX), ascorbate oxidase (AO), and alternative oxidase (AOX)] and scavenging [superoxide dismutase (SOD), catalase (CAT), peroxidase(POD), ascorbate peroxidase (APX), glutathione peroxidase (GPX), and glutathione reductase (GR)] antioxidant enzymes and antioxidant molecules like ascorbate, glutathione, and tocopherols (Considine and Foyer, 2014). In addition, redox state is regulated by various sugars and amino acids, which apart from playing role in plant metabolism are now considered an integral part of ROS scavenging machinery (Bolouri-Moghaddam et al., 2010; Hayat et al., 2012; Matros et al., 2015).

\section{REDOX REGULATION OF PLANT SEXUAL REPRODUCTION}

In flowering plants, flower is a seat of plant sexual reproduction encapsulating both male and female reproductive organs. The sterile part of flower consists of calyx and corolla, and the fertile part consists of androecium (male) and gynoecium (female). The detailed structure of plant reproductive organs and the sequence of events leading to fertilization are described in the legend of Figure 1. Starting from male or female gametogenesis through meiosis, followed by pollen/embryo sac growth, pollen-pistil interaction, and double-fertilization-the entire process is redox regulated.

Specific ROS levels, antioxidant molecules, and enzyme activities control the individual steps of sexual reproduction (Figure 1). Arabidopsis roxy1 mutant, deficient in CC-type GRXs (glutaredoxins: glutathione dependent oxidoreductases) displays reduced petal number and male-sterile phenotype. A deeper analysis revealed that this mutant lacks ability to enter meiotic phase (Xing and Zachgo, 2008). Furthermore, generation of reductive environment through exogenous supplementation of KI rescued the mscal (male sterile converted anther1: a CCtype GRX) mutant phenotype of maize, confirming that excess ROS formation was mainly responsible for male sterility (Kelliher and Walbot, 2012). Arabidopsis phytoalexin-deficient (pad2-1) mutant which is impaired in glutathione (GSH) synthesis shows poor pollen germination, indicating that GSH is essential for pollen development (Zechmann et al., 2011). Also, proper ROS gradient is required for pollen tube elongation. The rbohH and rbohJ (respiratory burst oxidase homolog $\mathrm{H}$ and $\mathrm{J}$ ) double mutant of Arabidopsis, which lacks the ability of ROS burst shows severe reduction in pollen tube tip growth (Kaya et al., 2014). Similarly, ROS homeostasis is important for female gametophyte development, where mitochondrial SOD (MSD1) plays a crucial role in regulating ROS levels (Martin et al., 2013). The mee33 (maternal effect embryo arrest 33) mutant lacking MSD1 activity shows defects in embryo sac development. The sperm cell release from pollen tube is a redox (ROS) dependent process. It is regulated through FERONIA (FER) receptor kinase which induces the production of high ROS levels, especially hydroxyl radicals, at the entrance of female gametophyte to facilitate the rupture of pollen tube in a calcium dependent manner (Duan et al., 2014). Interaction between pollen and pistil during pollination is one of a key step that determines the fate of fertilization, and decides whether seed set will happen or not. ROS $\left(\mathrm{H}_{2} \mathrm{O}_{2}\right)$ and $\mathrm{NO}$ (nitric oxide) mediated redox signaling is involved in pollen-pistil interactions (Sharma and Bhatla, 2013). Before pollen-pistil interaction, higher $\mathrm{H}_{2} \mathrm{O}_{2}$ production and enhanced activity of a Stigma-Specific Peroxidase (SSP) was observed at the receptive papillae, while pollen still retain high NO levels (McInnis et al., 2006; Bright et al., 2009; Zafra et al., 2010). After pollen landing on stigma, during the period of pollen-pistil interaction, the levels of $\mathrm{H}_{2} \mathrm{O}_{2}$ and activity of SSP declines dramatically in the stigmatic papillae. Following successful fertilization, formation of an embryo, and the development of mature seed requires extensive cell division and cell expansion, and both these processes are again redox regulated, and require low molecular weight antioxidants such as ascorbate and glutathione (Cairns et al., 2006; Livanos et al., 2012; Gallie, 2013). Thus, well-coordinated changes in the redox metabolism is essential for successful plant sexual reproduction. Such concept has also been proposed for other organisms such as Caenorhabditis elegans (De Henau et al., 2015) and mammals (Ufer et al., 2010).

\section{ABIOTIC STRESS INDUCES REDOX IMBALANCE DURING REPRODUCTIVE GROWTH}

Exposure of plants to abiotic stresses such as temperature fluctuations, water supply, and salinity, result in the formation of ROS (Gill and Tuteja, 2010; Suzuki et al., 2012; Zinta et al., 2014). Although, ROS acts as a signaling molecule at lower concentrations, it's accumulation beyond threshold leads to oxidative damage of macromolecules, resulting in growth retardation (Mittler, 2002). Because the success of plant reproductive development determines grain yield, it is obvious that stress exposure during reproductive phase will reduce the crop yield (Dolferus et al., 2011; Sage et al., 2015). Therefore, it is essential to understand how stress affects redox homeostasis in the context of plant sexual reproduction.

Stress negatively affects male reproductive development in plants (De Storme and Geelen, 2014). Drought imposed to rice during anthesis resulted in generation of sterile pollen, due to higher ROS accumulation and lower expression of transcripts related to antioxidant enzymes (Selote and Khanna-Chopra, 2004; Nguyen et al., 2009). Similarly, a study performing the comparative analysis of anthers of a drought sensitive and tolerant rice lines (under drought stress) revealed sensitive line exhibiting higher malondialdehyde (MDA) content and lower activity of antioxidant enzymes (SOD, POD, and CAT) as compared to tolerant line (Fu et al., 2011). These studies indicate that stress-induced over accumulation of ROS leads to pollen 


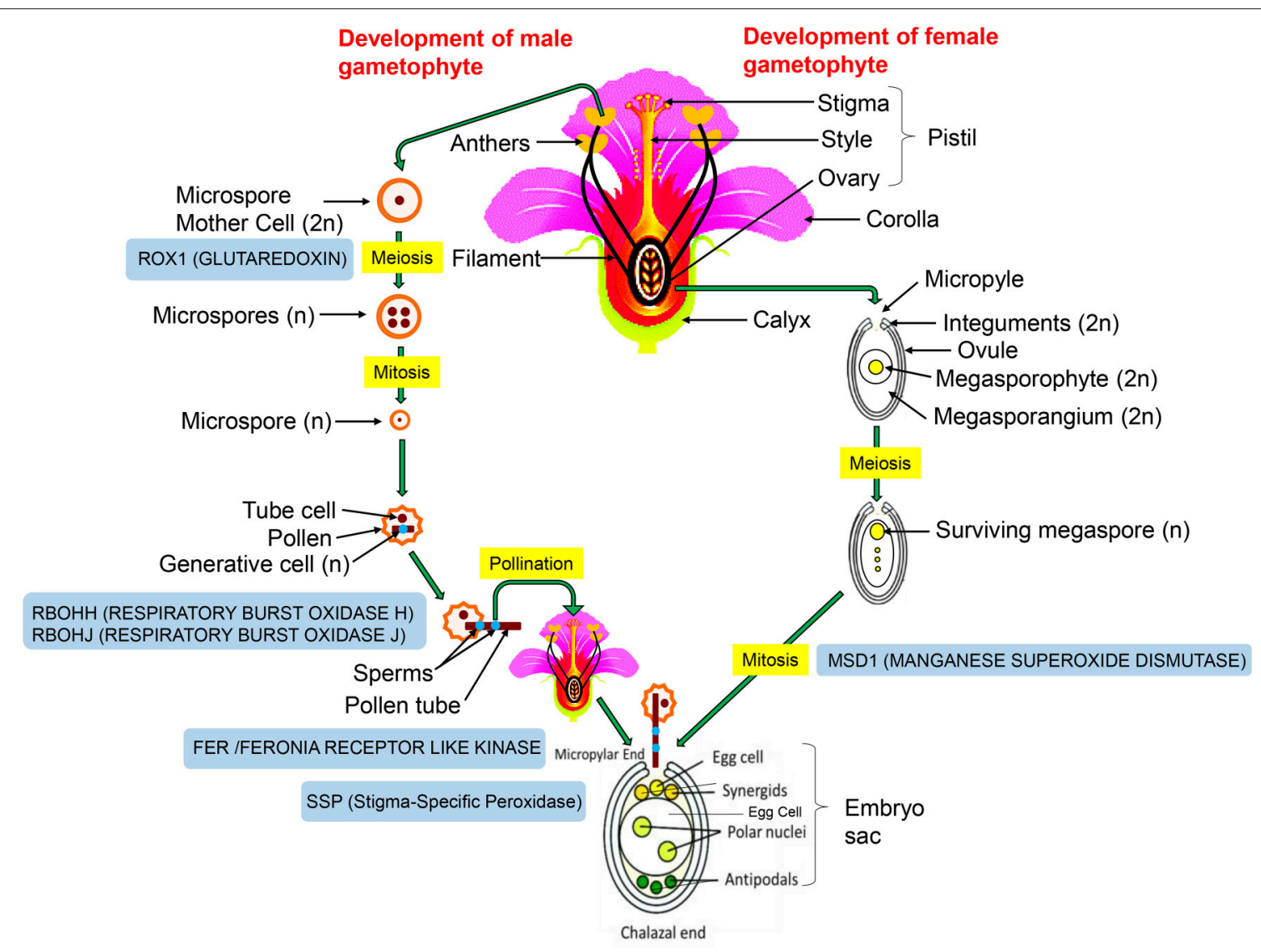

Pollination and Fertilization

FIGURE 1 | Structure of reproductive organs and the sequence of events involved in plant sexual reproduction. Male gametophyte (pollen grain) is comprised of a pollen tube consisting of a vegetative tube cell and two sperm cells. The female gametophyte (embryo sac) has two female gametes (egg and central cell) and accessory cells at the opposite poles. Two synergid cells adjoining the egg cell are located at micropylar entry of the ovule, while antipodal cells neighboring the central cell are present at the chalazal end. Sexual reproduction involves two major steps: pollination and fertilization. Pollination involves pollen-pistil interaction and fertilization involves fusion of meiotically generated haploid cells of a male (pollen grain) and a female gametophyte (embryo sac). During fertilization, pollen tube penetrates embryo sac at the micropyle by entering a synergid cell and delivers two sperm cells. Eventually, two gametic cell pairs of the embryo sac and two sperm cells undergo "double-fertilization." The fertilized central cell develops into endosperm, while the fertilized egg cell gives rise to embryo. The involvement of redox proteins at different steps of sexual reproduction is highlighted in blue boxes.

abortion and programmed cell death (PCD) of microspores in developing anthers, consequently resulting in male sterility.

Similarly, the female reproductive development is impaired by abiotic stress exposure (Moss and Downey, 1971; Sun et al., 2004; Loka and Oosterhuis, 2014). Heat stress caused activation of GR activity in the heat-stressed cotton pistils (Snider et al., 2009). Thermotolerant cotton variety showed higher pre-stress activity of SOD and GR in the pistil, suggesting it as a thermotolerance mechanism specific to female reproductive organs (Snider et al., 2011). Moreover, in salt stressed Arabidopsis plants, genes encoding ROS detoxifying enzymes, APX, and POD, were downregulated after ovules committed to abort. These changes in gene expression coincided with the accumulation of ROS in female gametophytes (Sun et al., 2005). This may have resulted from increased ROS biosynthesis, reduced ROS scavenging capacity or both, indicating that like male gametophyte, female gametophyte is also susceptible to stress-induced ROS accumulation.

Sugars or sugar-mediated transcriptional control play an important role in ROS homeostasis in the reproductive organs during stress episode (Couée et al., 2006; Keunen et al., 2013). Reduction in starch content prior to anthesis and decline in total soluble sugar content in mature pollen grains resulted in decreased pollen viability in tomato plants exposed to high temperature (Pressman et al., 2002). Recent metabolomic and transcriptomic profiling of floral organs (anthers and pistil) of heat-tolerant (N22) and heat-sensitive (Moroberekan) rice cultivars identified modulation in sugar metabolism as a regulatory mechanism imparting heat and drought tolerance to floral organs (Li et al., 2015). Therefore, stress-induced redox imbalance due to inefficient antioxidant system or alterations in the sugar metabolism could lead to higher ROS accumulation, 
which results in PCD of developing microspores/megaspores, finally leading to male sterility or ovule abortion.

\section{ENGINEERING REDOX COMPONENTS FOR IMPROVED REPRODUCTIVE SUCCESS}

A dynamic network of redox homeostasis related genes function to repair or abrogate stress-induced oxidative damage in the plant reproductive tissues (Meyer et al., 2012). Therefore, engineering redox components could be a way forward to impart stress tolerance in plants during reproductive phase.

The role of plant GRXs as master regulators of redox homeostasis during anther and gamete formation has been welldemonstrated (Kelliher and Walbot, 2012). A conserved plant specific CBSX (single cystathionine $\beta$-synthase domain) CCtype GRXs (ROXY1 and ROXY 2) genes and SQUAMOSA PROMOTER BINDING PROTEIN (SBP-box) transcription factors (target of miR156 and miR157) have been implicated in redox clean up during male reproductive development (Yoo et al., 2011). The role of these intriguing proteins was revealed in the studies using maize mscal mutant (Chaubal et al., 2003; Timofejeva et al., 2013). The CC-type GRXs, ROXY 1 and 2 act via interaction with basic leuine-zipper transcription factors, TGACG (TGA) motif-binding proteins TGA9 and TGA10 (Murmu et al., 2010). This association was demonstrated later in rice, where authors showed that rice MICROSPORELESS1(MIL1) gene, coding for a similar CC-type GRX, functions in the formation of surrounding somatic layer of anthers and in the transition of microsporocytes from mitosis to meiosis (Hong et al., 2012).

The role of cytosolic ascorbate peroxidase 2 (APX2) in enhanced seed production subjected to chronic heat stress was illustrated in Arabidopsis thaliana (Suzuki et al., 2013). Similarly, heat-stressed microspores in tomato showed upregulation of ROS-scavenging SIAPX3, safeguarding spores against toxic ROS accumulation (Frank et al., 2009). These two are relevant findings in the context of global warming, and demonstrate the protective role of APX against heat-induced oxidative damage in reproductive tissues.

One of plant's remarkable strategy to deal with stress is to escape the stressful environment via reproduction. Hence, proper timing of transition from vegetative to reproductive phase determines the success of reproduction. The OXS2 (OXIDATIVE STRESS 2) is a member of zinc-finger transcription factor family, which is involved in maintaining vegetative growth, stress tolerance, and stress-induced reproduction (Blanvillain et al., 2011). OXS2 is a stress responsive nucleo-cytoplasmic protein, shuttling between cytoplasm (no stress) to nucleus under stress conditions promoting stress resilience. OXS2 autoactivates itself, while coherently activating other floral integrator genes via direct binding to floral integrator promoter SUPPRESOR
OF CONSTANS (SOC1). This auto-regulatory loop of OXS2 may constitute an altruistic response choosing between stress tolerance to stress escape via reproduction (Blanvillain et al., 2011).

Two contrasting hypothesis have been proposed to explain the effects of redox status on meiosis and germline formation. The "reductive hypothesis" proposes that reduced ROS levels are required for successful meiosis (Kelliher and Walbot, 2014). This is supported by the evidences where chemical (Kelliher and Walbot, 2012) or genetic (Zechmann et al., 2011) approaches were used to reduce ROS levels, resulting in improved reproductive success as well as crop yield. In contrast, the "oxidative hypothesis" advocates increased ROS levels as a prerequisite for initiation of meiosis and sexual reproduction (Hörandl and Hadacek, 2013). In this view, the role of a meiotic protein SPO11 has been proposed. This protein initiates generation of DSBs (double-strand DNA breaks) during meiotic recombination and have antioxidant like properties to repair oxidatively damaged DNA (Hörandl and Hadacek, 2013). Moreover, oxidative stress can deregulate the epigenetic machinery comprising of DNA methylation and small RNAbased transcriptional regulation (Schmidt et al., 2015; Zhang et al., 2015). Thus, plant sexual reproduction is a highly complicated process which requires a fine-tuning of oxidativereductive (redox) pathways as well as epigenetic mechanisms, hence complicating the engineering of modules for enhancing stress tolerance in plants.

\section{CONCLUSIONS}

The redox control of plant reproductive development has been the long standing dilemma of plant scientists. Understanding the reproductive cost of oxidative stress requires a targeted molecular approach for engineering transgenes responsible for stress resilience in plant's reproductive system. But our knowledge is demarcated by methods to intertwine regulatory networks underlying reproductive development. Although much of the studies now focus on Arabidopsis as a model organism, future research should input judicious use of major crop species ensuring yield robustness in meeting continual demands of an ever increasing human population.

\section{AUTHOR CONTRIBUTIONS}

GZ and AK conceived the idea and outline for the prescribed article and thereafter compiled and incorporated the different sections contributed by co-authors. All the authors read and approved the final version before submission.

\section{ACKNOWLEDGMENTS}

GZ acknowledges support from Methusalem Funding to the Centre of Excellence "PLECO," University of Antwerp. 


\section{REFERENCES}

Bartoli, C. G., Casalongue, C. A., Simontacchi, M., Marquez-Garcia, B., and Foyer, C. H. (2013). Interactions between hormone and redox signalling pathways in the control of growth and cross tolerance to stress. Environ. Exp. Bot. 94, 73-88. doi: 10.1016/j.envexpbot.2012.05.003

Blanvillain, R., Wei, S., Wei, P., Kim, J. H., and Ow, D. W. (2011). Stress tolerance to stress escape in plants: role of the OXS2 zinc-finger transcription factor family. EMBO J. 30, 3812-3822. doi: 10.1038/emboj. 2011.270

Bolouri-Moghaddam, M. R., Le Roy, K., Xiang, L., Rolland, F., and Van Den Ende, W. (2010). Sugar signalling and antioxidant network connections in plant cells. FEBS J. 277, 2022-2037. doi: 10.1111/j.1742-4658.2010.07633.x

Bright, J., Hiscock, S. J., James, P. E., and Hancock, J. T. (2009). Pollen generates nitric oxide and nitrite: a possible link to pollen-induced allergic responses. Plant Physiol. Biochem. 47, 49-55. doi: 10.1016/j.plaphy.2008.09.005

Cairns, N. G., Pasternak, M., Wachter, A., Cobbett, C. S., and Meyer, A. J. (2006). Maturation of Arabidopsis seeds is dependent on glutathione biosynthesis within the embryo. Plant Physiol. 141, 446-455. doi: 10.1104/pp.106. 077982

Chaubal, R., Anderson, J. R., Trimnell, M. R., Fox, T. W., Albertsen, M. C., and Bedinger, P. (2003). The transformation of anthers in the mscal mutant of maize. Planta 216, 778-788. doi: 10.1007/s00425-002-0929-8

Considine, M. J., and Foyer, C. H. (2014). Redox regulation of plant development. Antioxid. Redox Signal. 21, 1305-1326. doi: 10.1089/ars.2013.5665

Couée, I., Sulmon, C., Gouesbet, G., and El Amrani, A. (2006). Involvement of soluble sugars in reactive oxygen species balance and responses to oxidative stress in plants. J. Exp. Bot. 57, 449-459. doi: 10.1093/jxb/erj027

De Henau, S., Tilleman, L., Vangheel, M., Luyckx, E., Trashin, S., Pauwels, M., et al. (2015). A redox signalling globin is essential for reproduction in Caenorhabditis elegans. Nat. Commun. 6, 8782. doi: 10.1038/ncomms 9782

De Storme, N., and Geelen, D. (2014). The impact of environmental stress on male reproductive development in plants: biological processes and molecular mechanisms. Plant Cell Environ. 37, 1-18. doi: 10.1111/pce.12142

Dolferus, R., Ji, X., and Richards, R. A. (2011). Abiotic stress and control of grain number in cereals. Plant Sci. 181, 331-341. doi: 10.1016/j.plantsci.2011.05.015

Duan, Q., Kita, D., Johnson, E. A., Aggarwal, M., Gates, L., Wu, H. M., et al. (2014). Reactive oxygen species mediate pollen tube rupture to release sperm for fertilization in Arabidopsis. Nat. Commun. 5, 3129. doi: 10.1038/ncomms4129

Frank, G., Pressman, E., Ophir, R., Althan, L., Shaked, R., Freedman, M., et al. (2009). Transcriptional profiling of maturing tomato (Solanum lycopersicum L.) microspores reveals the involvement of heat shock proteins, ROS scavengers, hormones, and sugars in the heat stress response. J. Exp. Bot. 60, 3891-3908. doi: $10.1093 / \mathrm{jxb} / \mathrm{erp} 234$

Fu, G.-F., Jian, S., Xiong, J., Li, Y.-R., Chen, H.-Z., Le, M.-K., et al. (2011). Changes of oxidative stress and soluble sugar in anthers involve in rice pollen abortion under drought stress. Agric. Sci. China 10, 1016-1025. doi: 10.1016/S16712927(11)60089-8

Gallie, D. R. (2013). L-ascorbic Acid: a multifunctional molecule supporting plant growth and development. Scientifica 2013:795964. doi: 10.1155/2013/795964

Gill, S. S., and Tuteja, N. (2010). Reactive oxygen species and antioxidant machinery in abiotic stress tolerance in crop plants. Plant Physiol. Biochem. 48, 909-930. doi: 10.1016/j.plaphy.2010.08.016

Gilroy, S., Suzuki, N., Miller, G., Choi, W. G., Toyota, M., Devireddy, A. R., et al. (2014). A tidal wave of signals: calcium and ROS at the forefront of rapid systemic signaling. Trends Plant Sci. 19, 623-630. doi: 10.1016/j.tplants.2014.06.013

Hayat, S., Hayat, Q., Alyemeni, M. N., Wani, A. S., Pichtel, J., and Ahmad, A. (2012). Role of proline under changing environments: a review. Plant Signal. Behav. 7, 1456-1466. doi: 10.4161/psb.21949

Hong, L., Tang, D., Shen, Y., Hu, Q., Wang, K., Li, M., et al. (2012). MIL2 (MICROSPORELESS2) regulates early cell differentiation in the rice anther. New Phytol. 196, 402-413. doi: 10.1111/j.1469-8137.2012.04270.x

Hörandl, E., and Hadacek, F. (2013). The oxidative damage initiation hypothesis for meiosis. Plant Reprod. 26, 351-367. doi: 10.1007/s00497-013-0234-7

Huijser, P., and Schmid, M. (2011). The control of developmental phase transitions in plants. Development 138, 4117-4129. doi: 10.1242/dev.063511
Kaya, H., Nakajima, R., Iwano, M., Kanaoka, M. M., Kimura, S., Takeda, S., et al. (2014). Ca2+-activated reactive oxygen species production by Arabidopsis $\mathrm{RbohH}$ and RbohJ is essential for proper pollen tube tip growth. Plant Cell 26, 1069-1080. doi: 10.1105/tpc.113.120642

Kelliher, T., and Walbot, V. (2012). Hypoxia triggers meiotic fate acquisition in maize. Science 337, 345-348. doi: 10.1126/science. 1220080

Kelliher, T., and Walbot, V. (2014). Maize germinal cell initials accommodate hypoxia and precociously express meiotic genes. Plant J. 77, 639-652. doi: $10.1111 /$ tpj.12414

Keunen, E., Peshev, D., Vangronsveld, J., Van Den Ende, W., and Cuypers, A. (2013). Plant sugars are crucial players in the oxidative challenge during abiotic stress: extending the traditional concept. Plant Cell Environ. 36, 1242-1255. doi: $10.1111 /$ pce.12061

Kocsy, G., Tari, I., Vanková, R., Zechmann, B., Gulyás, Z., Poór, P., et al. (2013). Redox control of plant growth and development. Plant Sci. 211, 77-91. doi: 10.1016/j.plantsci.2013.07.004

König, J., Muthuramalingam, M., and Dietz, K. J. (2012). Mechanisms and dynamics in the thiol/disulfide redox regulatory network: transmitters, sensors and targets. Curr. Opin. Plant Biol. 15, 261-268. doi: 10.1016/j.pbi.2011. 12.002

Li, X., Lawas, L. M., Malo, R., Glaubitz, U., Erban, A., Mauleon, R., et al. (2015). Metabolic and transcriptomic signatures of rice floral organs reveal sugar starvation as a factor in reproductive failure under heat and drought stress. Plant Cell Environ. 38, 2171-2192. doi: 10.1111/pce.12545

Livanos, P., Apostolakos, P., and Galatis, B. (2012). Plant cell division: ROS homeostasis is required. Plant Signal. Behav. 7, 771-778. doi: $10.4161 / \mathrm{psb} .20530$

Loka, D., and Oosterhuis, D. (2014). Water-deficit stress effects on pistil biochemistry and leaf physiology in cotton (Gossypium hirsutum, L.). South Afr. J. Bot. 93, 131-136. doi: 10.1016/j.sajb.2014.03.019

Martin, M. V., Fiol, D. F., Sundaresan, V., Zabaleta, E. J., and Pagnussat, G. C. (2013). Oiwa, a female gametophytic mutant impaired in a mitochondrial manganese-superoxide dismutase, reveals crucial roles for reactive oxygen species during embryo sac development and fertilization in Arabidopsis. Plant Cell 25, 1573-1591. doi: 10.1105/tpc.113.109306

Matros, A., Peshev, D., Peukert, M., Mock, H. P., and Van den Ende, W. (2015). Sugars as hydroxyl radical scavengers: proof-of-concept by studying the fate of sucralose in Arabidopsis. Plant J. 82, 822-839. doi: 10.1111/tpj.12853

McInnis, S. M., Desikan, R., Hancock, J. T., and Hiscock, S. J. (2006). Production of reactive oxygen species and reactive nitrogen species by angiosperm stigmas and pollen: potential signalling crosstalk? New Phytol. 172, 221-228. doi: 10.1111/j.1469-8137.2006.01875.x

Meyer, Y., Belin, C., Delorme-Hinoux, V., Reichheld, J.-P., and Riondet, C. (2012) Thioredoxin and glutaredoxin systems in plants: molecular mechanisms, crosstalks, and functional significance. Antioxid. Redox Signal. 17, 1124-1160. doi: 10.1089 /ars.2011.4327

Mittler, R. (2002). Oxidative stress, antioxidants and stress tolerance. Trends Plant Sci. 7, 405-410. doi: 10.1016/S1360-1385(02)02312-9

Moss, G., and Downey, L. (1971). Influence of drought stress on female gametophyte development in corn (Zea mays L.) and subsequent grain yield. Crop Sci. 11, 368-372. doi: 10.2135/cropsci1971.0011183X0011000 $30017 \mathrm{x}$

Murmu, J., Bush, M. J., Delong, C., Li, S., Xu, M., Khan, M., et al. (2010). Arabidopsis basic leucine-zipper transcription factors TGA9 and TGA10 interact with floral glutaredoxins ROXY1 and ROXY2 and are redundantly required for anther development. Plant Physiol. 154, 1492-1504. doi: 10.1104/pp.110.159111

Nguyen, G., Hailstones, D., Wilkes, M., and Sutton, B. (2009). Drought-induced oxidative conditions in rice anthers leading to a programmed cell death and pollen abortion*. J. Agron. Crop Sci. 195, 157-164. doi: 10.1111/j.1439037X.2008.00357.x

Pressman, E., Peet, M. M., and Pharr, D. M. (2002). The effect of heat stress on tomato pollen characteristics is associated with changes in carbohydrate concentration in the developing anthers. Ann. Bot. 90, 631-636. doi: $10.1093 / \mathrm{aob} / \mathrm{mcf} 240$

Sage, T. L., Bagha, S., Lundsgaard-Nielsen, V., Branch, H. A., Sultmanis, S., and Sage, R. F. (2015). The effect of high temperature stress on male 
and female reproduction in plants. Field Crops Res. 182, 30-42. doi: 10.1016/j.fcr.2015.06.011

Schmidt, A., Schmid, M. W., and Grossniklaus, U. (2015). Plant germline formation: commonconcepts and developmental flexibility in sexual and asexual reproduction. Development 142, 229-241. doi: 10.1242/dev.102103

Selote, D. S., and Khanna-Chopra, R. (2004). Drought-induced spikelet sterility is associated with an inefficient antioxidant defence in rice panicles. Physiol. Plant. 121, 462-471. doi: 10.1111/j.1399-3054.2004.00341.x

Sharma, B., and Bhatla, S. (2013). Accumulation and scavenging of reactive oxygen species and nitric oxide correlate with stigma maturation and pollenstigma interaction in sunflower. Acta Physiol. Plant. 35, 2777-2787. doi: 10.1007/s11738-013-1310-1

Snider, J. L., Oosterhuis, D. M., Skulman, B. W., and Kawakami, E. M. (2009). Heat stress-induced limitations to reproductive success in Gossypium hirsutum. Physiol. Plant. 137, 125-138. doi: 10.1111/j.1399-3054.2009.01266.x

Snider, J., Oosterhuis, D., and Kawakami, E. (2011). Mechanisms of reproductive thermotolerance in Gossypium hirsutum: the effect of genotype and exogenous calcium application. J. Agron. Crop Sci. 197, 228-236. doi: 10.1111/j.1439037X.2010.00457.x

Steinhorst, L., and Kudla, J. (2013). Calcium and reactive oxygen species rule the waves of signaling. Plant Physiol. 163, 471-485. doi: 10.1104/pp.113.222950

Sun, K., Cui, Y., and Hauser, B. A. (2005). Environmental stress alters genes expression and induces ovule abortion: reactive oxygen species appear as ovules commit to abort. Planta 222, 632-642. doi: 10.1007/s00425-005-0010-5

Sun, K., Hunt, K., and Hauser, B. A. (2004). Ovule abortion in Arabidopsis triggered by stress. Plant Physiol. 135, 2358-2367. doi: 10.1104/pp.104.043091

Suzuki, N., Koussevitzky, S., Mittler, R., and Miller, G. (2012). ROS and redox signalling in the response of plants to abiotic stress. Plant Cell Environ. 35, 259-270. doi: 10.1111/j.1365-3040.2011.02336.x

Suzuki, N., Miller, G., Sejima, H., Harper, J., and Mittler, R. (2013). Enhanced seed production under prolonged heat stress conditions in Arabidopsis thaliana plants deficient in cytosolic ascorbate peroxidase 2. J. Exp. Bot. 64, 253-263. doi: $10.1093 /$ jxb/ers335

Timofejeva, L., Skibbe, D. S., Lee, S., Golubovskaya, I., Wang, R., Harper, L., et al. (2013). Cytological characterization and allelism testing of anther developmental mutants identified in a screen of maize male sterile lines. G3 (Bethesda) 3, 231-249. doi: 10.1534/g3.112.004465

Traverso, J. A., Pulido, A., Rodríguez-García, M. I., and Alché, J. D. (2014). Thiol-based redox regulation in sexual plant reproduction: new insights and perspectives. Front Plant Sci. 4:465. doi: 10.3389/fpls.2013.00465
Ufer, C., Wang, C. C., Borchert, A., Heydeck, D., and Kuhn, H. (2010). Redox control in mammalian embryo development. Antioxid. Redox Signal. 13, 833-875. doi: 10.1089/ars.2009.3044

Xia, X. J., Zhou, Y. H., Shi, K., Zhou, J., Foyer, C. H., and Yu, J. Q. (2015). Interplay between reactive oxygen species and hormones in the control of plant development and stress tolerance. J. Exp. Bot. 66, 2839-2856. doi: 10.1093/jxb/erv089

Xing, S., and Zachgo, S. (2008). ROXY1 and ROXY2, two Arabidopsis glutaredoxin genes, are required for anther development. Plant J. 53, 790-801. doi: 10.1111/j.1365-313X.2007.03375.X

Yoo, K. S., Ok, S. H., Jeong, B.-C., Jung, K. W., Cui, M. H., Hyoung, S., et al. (2011). Single cystathionine $\beta$-synthase domain-containing proteins modulate development by regulating the thioredoxin system in Arabidopsis. Plant Cell 23, 3577-3594. doi: 10.1105/tpc.111.089847

Zafra, A., Rodríguez-García, M. I., and Alché, J. D. (2010). Cellular localization of ROS and NO in olive reproductive tissues during flower development. BMC Plant Biol. 10:36. doi: 10.1186/1471-2229-10-36

Zechmann, B., Koffler, B. E., and Russell, S. D. (2011). Glutathione synthesis is essential for pollen germination in vitro. BMC Plant Biol. 11:54. doi: 10.1186/1471-2229-11-54

Zhang, H., Xia, R., Meyers, B. C., and Walbot, V. (2015). Evolution, functions, and mysteries of plant ARGONAUTE proteins. Curr. Opin. Plant Biol. 27, 84-90. doi: 10.1016/j.pbi.2015.06.011

Zinta, G., Abdelgawad, H., Domagalska, M. A., Vergauwen, L., Knapen, D., Nijs, I., et al. (2014). Physiological, biochemical, and genome-wide transcriptional analysis reveals that elevated $\mathrm{CO} 2$ mitigates the impact of combined heat wave and drought stress in Arabidopsis thaliana at multiple organizational levels. Glob. Chang. Biol. 20, 3670-3685. doi: 10.1111/gcb.12626

Conflict of Interest Statement: The authors declare that the research was conducted in the absence of any commercial or financial relationships that could be construed as a potential conflict of interest.

Copyright (c) 2016 Zinta, Khan, AbdElgawad, Verma and Srivastava. This is an open-access article distributed under the terms of the Creative Commons Attribution License (CC BY). The use, distribution or reproduction in other forums is permitted, provided the original author(s) or licensor are credited and that the original publication in this journal is cited, in accordance with accepted academic practice. No use, distribution or reproduction is permitted which does not comply with these terms. 